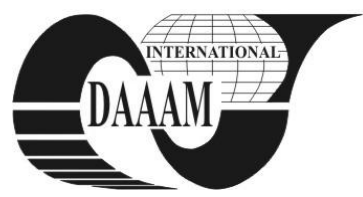

\title{
A (2,3)-PADE APPROXIMATION OF SECULAR FUNCTION OF TOEPLITZ MATRIX
}

\section{KOSTIC, A[leksandra] \& VELIC, M[elisa]}

\begin{abstract}
In this note we present a (2,3)-Padé approximation of secular function for computing the smallest eigenvalue $\lambda_{1}$ of a real symmetric positive definite Toeplitz matrix (RSPDTM). This method is based on approximation of secular function by using the Taylor series characteristic polynomial of $R$ SPDTM The characteristic polynomial has been approximated with the polynomial of the third order from the Taylor series because it is easy to calculate $p_{n-1}^{\prime \prime}(\lambda)$ from specific structure of Toeplitz matrix from Gohberg-Simencul formulae. It is very easy to calculate $p_{n-1}^{\prime \prime \prime}(\lambda)$ from the secular function.
\end{abstract}

Key words: eigenvalue problem, Toeplitz matrix, (2,3)-Padé approximation

\section{INTRODUCTION}

From Pisarenko's work (Pisarenko, 1973) the problem of finding the smallest eigenvalue of a real symmetric, positive definite Toeplitz matrix (RSPDTM) plays an important role in signal processing. The computation of the minimum eigenvalue of $T_{n}$ was studied in, e. g. in (Cybenko \& Van Loan, 1986; Kostic, 2004; Kostic \& Cohodar, 2008; Mackens \& Voss, 1997, 2000; Melman, 2006; Mastronardi \& Boley, 1999).

In this paper we propose a (2,3)-Padé approximation for the computation of the root of the secular function of a real symmetric positive definite Toeplitz matrix. This method is a combination of approximation for secular function and approximation for characteristic polynomial.

The paper is organized as follows. In Section 2 we present the basic properties of Toeplitz matrices and the notation we will use. In Section 3 we present (2,3)-Padé approximation for secular function. In Section 4 we present numerical results for Section 3. In Section 5 we present conclusion of the paper.

\section{PRELIMINARIES}

The Teoplitz matrix is quadratic matrix which has the same elements in its respective diagonals, which means that $a_{i j}=$ $a_{n+1-j, n+1-i}$. Because we take in consideration symmetric matrices it also means that $a_{i j}=a_{j i}$. The Teoplitz matrix is defined with vector $\left(1, t_{1}, \ldots, t_{n-1}\right) \in R^{n}$ so the $(i, j)^{\text {th }}$ element of an $n \times n$ symmetric Toeplitz matrix $T_{n}$ is given by $t_{|i-j|}$. We can conclude, form above given, that Teoplitz matrices are centrosymmetric and satisfy $J T_{n} J=T_{n}$. We use $I$ for the identity matrix and $J:=\left(\delta_{i, n+1-i}\right)_{i, j=1, \ldots, n}$ for the exchange, or "flip" matrix.

\section{A (2,3)-PADÉ APPROXIMATION}

A Padé rational approximation to $\mathrm{f}(\mathrm{x})$ on $[\mathrm{a}, \mathrm{b}]$ is the quotient of two polynomials $P_{n}(x)$ and $Q_{m}(x)$ of degrees $\mathrm{n}$ and $\mathrm{m}$, respectively. We use the notation $R_{n, m}(x)$ to denote this quotient:

$$
R_{n, m}(x)=\frac{P_{n}(x)}{Q_{m}(x)}
$$

Padé approximation is based on theorem Theorem (Padé Approximation)

Assume that $f \in C^{n+m}$, and that $f(x)$ Maclaurin polynomial expansion of degree at least $n+m$. The

$$
f(x) \approx R_{n, m}(x)=\frac{P_{n}(x)}{Q_{m}(x)}
$$

where $P_{n}(x)$ and $Q_{m}(x)$ are polynomials of degree $n$ and $m$, respectively.

Melman (Melman 1999) considered rational approximations

$$
r_{j}(\lambda)=-1+\lambda+\rho_{j}(\lambda)
$$

of $f$ where

$$
\rho_{1}(\lambda):=\frac{a}{b-\lambda}, \quad \rho_{2}(\lambda):=a+\frac{b}{c-\lambda}, \rho_{3}(\lambda):=\frac{a}{b-\lambda}+\frac{c}{d-\lambda}
$$

And the parameters $a, b, c, d$ are determined such that $\rho_{j}^{(k)}(0)=\left.\frac{d^{k}}{d \lambda^{k}} t^{T}\left(T_{n-1}-\lambda I\right)^{-1} t\right|_{\lambda=0}=k ! t^{T} T_{n-1}^{-(k+1)} t, k=0,1, \ldots, j \cdot$

Thus $\rho_{1}, \rho_{2}$ and $\rho_{3}$, respectively, are the $(0,1)-,(1,1)-$ and $(1,2)-$ Padé approximations of $\Phi(\lambda):=t^{T}\left(T_{n-1}-\lambda I\right)^{-1} t$ (Melman, 1999).

Here we present a new rational approximation of the secular function. This new rational approximation is actually $(2,3)$ Padé approximation for secular function.

The secular function $q(\lambda)=-1+\lambda+t^{T}\left(T_{n-1}-\lambda I\right)^{-1} t$ may be written as

$$
q(\lambda)=-\frac{p_{n}(\lambda)}{p_{n-1}(\lambda)}
$$

where $p_{n}(\lambda)$ and $p_{n-1}(\lambda)$ are characteristic polynomials of $T_{n}$ and $T_{n-1}$ respectively.

We consider a rational approximation

$$
r(\lambda)=d+\lambda-\bar{\lambda}+\frac{A_{2}(\lambda-\bar{\lambda})^{2}+A_{1}(\lambda-\bar{\lambda})+A_{0}}{B_{3}(\lambda-\bar{\lambda})^{3}+B_{2}(\lambda-\bar{\lambda})^{2}+B_{1}(\lambda-\bar{\lambda})+B_{0}}
$$

of $q$, where $d:=\bar{\lambda}-1$ and parameters $A_{2}$ and $B_{3}$ such that

$$
\begin{aligned}
& r(\overline{\bar{\lambda}})=q(\overline{\bar{\lambda}}) \\
& r^{\prime}(\overline{\bar{\lambda}})=q^{\prime}(\overline{\bar{\lambda}})
\end{aligned}
$$

$\bar{\lambda}$ and $\overline{\bar{\lambda}}$ are not in the spectrum of $T_{n}$ and $T_{n-1}$. Parameters $\mathrm{B}_{0}, \mathrm{~B}_{1}$ and $\mathrm{B}_{3}$ are given by to approximate characteristic 
polynomial of $T_{n-1}$ with the polynomial of the second order based on the Taylor series. Parameters $\mathrm{A}_{0}$ and $\mathrm{A}_{1}$ can be easily calculated by dividing characteristic polynomials $p_{n}$ and $p_{n-1}$. We give

$$
\begin{aligned}
& B_{0}=\frac{1}{q(\bar{\lambda})} \\
& B_{1}=-\frac{p_{n-1}^{\prime}(\bar{\lambda})}{p_{n-1}(\bar{\lambda})} \cdot \frac{1}{q(\bar{\lambda})} \\
& B_{2}=\frac{p_{n-1}^{\prime \prime}(\bar{\lambda})}{2 p_{n-1}(\bar{\lambda})} \cdot \frac{1}{q(\bar{\lambda})} \\
& A_{0}=1-\frac{d}{q(\bar{\lambda})}
\end{aligned}
$$

$$
A_{1}=-\frac{p_{n}^{\prime}(\bar{\lambda})}{p_{n}(\bar{\lambda})}-\frac{1}{q(\bar{\lambda})}+\frac{d}{q(\bar{\lambda})} \cdot \frac{p_{n-1}^{\prime}(\bar{\lambda})}{p_{n-1}(\bar{\lambda})}
$$$$
t=\overline{\bar{\lambda}}-\bar{\lambda}
$$$$
B_{3}=\frac{\left(B_{1} t+2 B_{0}\right) \cdot(q(\overline{\bar{\lambda}})-d-t)-\left(B_{1} t^{3}+B_{1} t^{2}+B_{0} t\right)\left(q^{\prime}(\overline{\bar{\lambda}})-1\right)-A_{1} t-}{t^{3} \cdot\left(q^{\prime}(\overline{\bar{\lambda}}) t+q(\overline{\bar{\lambda}})-d-2 t\right)}
$$$$
A_{2}=\frac{(q(\overline{\bar{\lambda}})-d-t)\left(B_{3} t^{3}+B_{2} t^{2}+B_{1} t+B_{0}\right)-A_{1} t-A_{0}}{t^{2}}
$$

For easy calculation of given parameters we need theoretical base given in works (Kostic 2009, Kostic \& Cohodar 2008 and Melman 2006).

\section{NUMERICAL RESULTS}

To test the method we considered the following class of Toeplitz matrices:

$$
T_{n}=m \sum_{k=1}^{n} \eta_{k} T_{2 \pi \theta_{k}}
$$

where $m$ is chosen such that the diagonal of $T_{n}$ is normalized to $t_{0}=1 . \quad T_{\theta}=\left(t_{i, j}\right)=(\cos (\theta(i-j)))$ and $\eta_{k}$ and $\theta_{k}$ are uniformly distributed random in the interval $[0,1]$ ( cf. Cybenko and Van Loan). Table 1 contains the average number of Durbin steps needed to determine in 100 test problem each of the dimension $n=32,64,128,256,512$ and 1024 .

\begin{tabular}{|c|c|}
\hline Dim & New method \\
\hline 32 & 4.8575 \\
\hline 64 & 5.29 \\
\hline 128 & 5.2925 \\
\hline 256 & 5.72 \\
\hline 512 & 6.38 \\
\hline 1024 & 6.8 \\
\hline
\end{tabular}

Tab. 1. The average number of Durbin steps

\section{CONCLUSION}

In this paper we present a (2,3)-Padé approximation of the secular function for computing the smallest eigenvalue $\lambda_{1}$ of a real symmetric positive definite Toeplitz matrix (RSPDTM) . This method is based on approximation of secular function by using the Taylor series characteristic polynomial of RSPDTM. In this way, we improved previously developed algorithm what we also have expected, because we used second derivative of characteristic polynomial. Calculation of second derivative of characteristic polynomial is interesting result witch enables new rational approximation for secular function. New rational approximation presented in this note is actually $(2,3)$ - Padé approximation. It is proven that the Padé approximation is the best approximation of a rational function. In this paper we have also numerically check this contention. This is the main contribution of the paper.

It is very interesting to approximate the even and odd secular functions on above pointed out manner but with much more using property of symmetry of the Toeplitz matrices. This will be further investigated in the next step of our future research plans.

\section{REFERENCES}

Cantoni A. \& Butler F. (1976) Eigenvalues and eigenvectors of symmetric centrosymmetric matrices, Linear Algebra Appl., 13 pp. 275-288. MR0395514(53:476)

Cybenko, G. \& Van Loan, C.F. (1986). Computing the minimum eigenvalue of a symmetric positive definite Toeplitz matrix, SIAM J. Sci. Stat. Comput, Vol. 7, No. 1, pp. 123-131. MRO819462(87b:65042)

Dembo, A. (1988)Bounds on the extreme eigenvalues of positive definite Toeplitz matrices. IEEE Trans. Inform. Theory, 34, .352-355

Gohberg, I.C. \& Semencul, A. A. (1972). The inversion of finite Toeplitz matrices and their continual analogues. (Russian), Mat. Issued. Vol. 7., No. 2(24), pp. 201-223. MR0353038 (50:5524)

Kostic A. (2004) Verfahren zur Bestimmung einiger extremaler Eienwerte einer symmetrischen Toeplitz Matrix, Shaker Verlag, ISBN 3-8322-3235-4, Aachen

Kostic A.(2009) Approximation of characteristic polynomial of SPDTM to appear in DAAAM International scientific book 2009.

Kostic A. \& Cohodar M. (2008). Quadratic approximation of characteristic polynomial of symmetric positive definite Toeplitz matrix, Proceedings of 19th International DAAAM Symposium, ISBN 978-3-901509-68-1,ISSN 1726-9679, pp 192

Mackens , W. \& Voss, H. (2000). Computing the minimum eigenvalue of a symmetric positive definite Toeplitz matrix by Newton-type methods, SIAM J. Sci. Comput., Vol. 21. No. 4, pp. 1650-1656 MR1756049 (2001g:65043)

Mackens , W. \& Voss, H. (1997). The minimum eigenvalue of a symmetric positive definite Toeplitz matrix and rational Hermitian interpolation, SIAM J. Matr. Anal. Appl, Vol. 18. pp. 523-534

Mastronardi, N \& Boley, D.(1999). Computing the smallest eigenpair of a symmetric positive definite Toeplitz matrix, SIAM J. Sci. Comput., Vol. 20, No. 5, pp. 1921-1927. MR1694690

Melman, A. (1999), Bounds on the extreme eigenvalues of real symmetric Teoplitz matrices, SIAM J. Matrix Anal. Appl., 21 (2):362-378

Melman, A. (2003), Computation of the smallest even and odd eigenvalues of symmetric positive indefinite Toplitz matrix. SIAM J. Matr. Anal. Appl.

Melman, A. (2006). Computation of the Newton step for the even and odd characteristic polynomials of a symmetric positive definite Toeplitz matrix, Mathematics of computation, Vol. 75, No 254, pp. 817-832, S 0255718(05)01796-5

Pisarenko, V. F. (1973). The retrieval of harmonics from a covariance function. Geophys, J. R. astr. Soc., Vol. 33., 347-366

Voss, H. (1999). Symmetric schemes for computing the minimum eigenvalue of a symmetric Toeplitz matrix, Lin. Alg. Appl., 287: 377-385

Voss, H. (1999). Bounds for the minimum eigenvalue of symmetric Toeplitz matrix, Electronic Transactions on Numerical Analysis, Vol. 8, 127-138 